\title{
Serum KL-6 as predictive and prognostic marker of interstitial lung disease in childhood connective tissue diseases: a pilot study
}

\author{
R. El-Beheidy ${ }^{1}$, A.M. Domouky ${ }^{1,2}$, H. Zidan³, Y.A. Amer ${ }^{4}$ \\ ${ }^{1}$ Pulmonology, Immunology and Allergy Unit, Pediatrics Department, Faculty of Medicine, Zagazig University, Egypt; \\ ${ }^{2}$ Human Anatomy \& Embryology Department, Faculty of Medicine, Zagazig University, Egypt; \\ ${ }^{3}$ Medical Biochemistry and Molecular Biology, Faculty of Medicine, Zagazig University, Egypt; \\ ${ }^{4}$ Rheumatology \& Rehabilitation Department, Faculty of Medicine, Zagazig University, Egypt
}

\begin{abstract}
This study was aimed to evaluate serum KL-6 levels to determine if this marker can be used for diagnosing and assessing severity of interstitial lung disease (ILD) in children with connective tissue disorders.

In total, 40 patients [18 patients with juvenile systemic lupus erythematosus (JSLE), 10 patients with juvenile idiopathic arthritis (JIA), 8 patients with juvenile mixed connective tissue disease (JMCTD), 3 patients with juvenile systemic sclerosis (JSSc), and 1 patient with juvenile dermatomyositis (JDM)] and 20 healthy controls were included in this study. Age, sex, and duration of CTD and ILD (if any) were recorded. Blood samples from all the patients and controls were examined by ELISA.

20 of the 40 patients with CTD $(50 \%)$ had ILD, 12 were mild and 8 were severe as assessed by spirometry. The median serum KL-6 level was 102.7 U/mL (76.1-180.8) in the CTD with severe ILD group, 72.2 U/mL (58.4$100.5)$ in the CTD with mild ILD group, $56.7 \mathrm{U} / \mathrm{mL}$ (35.8-68.5) in the CTD without ILD group, and $52.3 \mathrm{U} / \mathrm{mL}$ (32.8-62.4) in the control group. KL-6 levels were significantly higher in the CTD with ILD ( $<<0.05)$, at a cutoff of $63.4 \mathrm{U} / \mathrm{ml}$ identified by ROC curve, serum KL-6 showed a sensitivity of $95.2 \%$ and specificity of $89.7 \%$. KL-6 is a valuable biomarker for diagnostic purposes and to detect severity in ILD in childhood CTD.
\end{abstract}

SUMMARY

Key words: Interstitial lung diseases, connective tissue diseases, KL-6, pulmonary function test.

Reumatismo, 2021; 73 (3): 147-155

\section{INTRODUCTION}

Tuvenile rheumatoid arthritis (JRA), juvenile systemic lupus erythematosus (JSLE), juvenile systemic sclerosis (JSSc), juvenile dermatomyositis (JDM), and juvenile mixed connective tissue disease (JMCTD) are a dissimilar group of systemic inflammatory diseases characterized by the presence of circulating autoantibodies and autoimmune-mediated multiorgan system involvement (1-4). Pediatric onset of these disorders causes diagnostic stress for the clinician due to the probable years of disease burden and complications to be expected (5). The lungs are a common target in CTD, and all components of the respiratory system are at risk. Diffuse lung disease (DLD) in children with rheumatologic disease rep- resents a pattern of pulmonary diseases as pleuritis, interstitial lung disease (ILD), thromboembolic disease, pulmonary hypertension, and pulmonary hemorrhage secondary to vasculitis. In this cases, complex care and monitoring of disease activity and progression are compulsory for life (6).

Interstitial lung disease (ILD) represents a broad group of diffuse parenchymal lung injury patterns characterized by variable degrees of inflammation and fibrosis, which is a common manifestation of CTD and is a leading cause of significant morbidity and mortality. ILD may arise at any time over the course of CTD or may be the first manifestation of disease. ILD may be subclinical (radiographic or physiologic abnormalities without symptoms), chronically progressive, or may present in a fulminant, life-
Corresponding author: Ayat Mahmoud El-Domouky Lecturer at Human Anatomy \& Embryology Department, Researcher at Pulmonology, Immunology and Allergy Unit, Pediatrics Department, Faculty of Medicine, Zagazig University, Zagazig, Egypt

E-mail: amdomouky@medicine.zu.edu.eg; drayat_pedia@outlook.com 
threatening manner (7). The diagnosis of ILD necessitates meticulous evaluation of clinical, radiological, physiological, and pathological changes. Pulmonary function tests (PFTs) and radiological imaging may be used to detect $\operatorname{ILD}(8,9)$. Consequently, novel noninvasive procedures for early detection are essential.

KL-6 is a high molecular weight mucin-like protein produced by alveolar type- 2 pneumocytes. KL-6 is elevated in regenerated normal lung tissue, and when the air-blood barrier is disturbed in the lungs or expression of alveolar type- 2 pneumocytes is increased (10-12). KL-6 is suggested as a sensitive and specific marker of the development of ILD in adults and is also increased in cases of CTD with comorbid ILD (13-16). There are few studies to determine the relationship between KL-6 and CTD-ILD in children.

Therefore, our study aims to evaluate the correlation between serum KL-6 levels with both the presence of interstitial lung disease (ILD) and its severity in childhood connective tissue diseases for early detection and better management of interstitial lung disease in CTD children.

\section{PATIENTS AND METHODS}

\section{Study population}

CTD children were selected from the medical record database between October 2019 and April 2020 from Rheumatology and Rehabilitation Department, and Pediatric department; Pulmonology, Immunology and Allergy Unit, faculty of medicine, Zagazig University. Written informed consent was obtained from all participants. The study was done according to the Code of Ethics of the World Medical Association (Declaration of Helsinki) for studies involving humans, and approved accordance with the guidelines of the Institutional Review Board (IRB) of the Faculty of Medicine, Zagazig University, Egypt (Approval No.: ZU-IRB \#544459/147-2019). Out of 40 CTD children, 16 were already diagnosed with interstitial lung disease, and 4 patients were newly diagnosed.

The study included 20 patients with CTD without ILD, 20 patients with CTD-ILD and 20 healthy control children attending the outpatient clinic. They all had a similar age and sex distribution. In total, the study included children with connective tissue diseases aged 5-15 years at diagnosis selected according to the classification criteria of the European League Against Rheumatism (EULAR) for rheumatoid arthritis (RA), 2010 (17), systemic lupus erythematosus (SLE), 2019 (18), dermatomyositis (DM), 2017 (19), and juvenile systemic sclerosis (SSc), 2007 (20). The selection was also based on the diagnostic criteria for mixed connective tissue disease (MCTD) from the Japan research committee of the ministry of health, labor, and welfare for systemic autoimmune diseases, 2019 (21). We excluded patients with an active malignancy, and active acute infections. History taking, general and local examinations were performed for all the children included.

\section{Diagnostic approach to ILD in CTD children}

In patients with respiratory symptoms (dry cough, rapid and/or difficult breathing, or exercise intolerance) and/or respiratory signs (tachypnea at rest, adventitious sounds, retractions, digital clubbing, failure to thrive, or respiratory failure) or even in asymptomatic patients with restrictive lung pattern on spirometry, the diagnosis of ILD required the following $(22,23)$ :

1) exclusion of other known causes of diffuse lung disease, infection (by CRB, ESR, sputum culture), cystic fibrosis (proven by sweat chloride test), primary vs secondary immunodeficiency (based on complete blood count and differential WBCs count, antibodies to HIV, vaccine response), gastroesophageal reflux (barium swallow study), congenital heart disease (shown by echocardiography) (23).

2) The presence of the pattern of usual interstitial pneumonia (UIP) at High-Resolution Computed Tomography (HRCT).

3) Specific combinations of HRCT and histopathology patterns in patients subjected to lung tissue biopsy (22).

\section{Lung HRCT}

Patients were evaluated by lung HRCT. All patients were scanned from the apex to the 
basis of the lung by routine end-inspiratory spiral CT with a slice thickness of $5.0 \mathrm{~mm}$. High-resolution re-establishment was used to scan the patients twice for confirmation. Lung X-rays and HRCT were interpreted by both the radiologist and the pediatric pulmonologist to check whether any specific criteria of definite UIP were met, such as subpleural and basal predominant component, heterogeneous distribution, honeycombing with or without peripheral traction, or bronchiectasis).

\section{Surgical lung biopsy}

SLB was performed only for four patients who had a non-specific HRCT in spite of their clear clinical manifestations and restricted pulmonary function. In these four cases, lung biopsy was completed with a mini thoracotomy. Two large lung samples were harvested from two separate portions using a chest tube inserted prior to stitching. Then these biopsy samples were assessed by histopathological examination. The lung histology patterns observed revealed a NonSpecific Interstitial Pneumonia (NSIP) in 3 patients, and a Desquamative Interstitial Pneumonia (DIP) in one patient. NSIP is characterized by mild to moderate interstitial chronic inflammation and type 2 alveolar epithelial cells hyperplasia in inflammation areas. DIP is characterized by airspaces filled with alveolar macrophages, thickened alveolar septa, scattered mixed inflammatory cells and minimal fibrosis.

\section{ASSESSMENT} OF ILD SEVERITY

\section{Spirometry}

Patients underwent the PFT using a Jeager Vyntus spirometry. The following parameters were recorded: forced expiratory volume in $1 \mathrm{~s}$ (FEV1), forced vital capacity (FVC), FEV1/FVC ratio, expressed as a percentage of measured value/predicted value. The interpretation of PFT according to Jiang et al. (24) was adopted, whereby FVC, FEV $1>80 \%$ of predicted values was considered as normal; FVC, FEV1 $<80 \%$ and $>60 \%$ of predicted values was considered as mild ILD Patients, and FVC, FEV1
$<60 \%$ of predicted values was considered as severe ILD Patients.

\section{6-minute walk test}

6MWT was performed in all participants according to Lancaster (25). Participants held pulse oximeters. Heart rate and oxygen saturation $(\mathrm{SpO} 2)$ were recorded before and immediately after the 6-minute walk. To assess the grade of dyspnea, participants were asked to rate their dyspnea using the modified Borg scale by choosing a score from 0 to 10 , with 0 being no significant dyspnea and 10 being highest grade of dyspnea.

\section{Laboratory tests}

Blood samples $(10 \mathrm{~mL})$ were drawn from all the patients and controls, and stored at $-80^{\circ} \mathrm{C}$ after centrifugation at $1000 \mathrm{rpm}$ for $15 \mathrm{~min}$. KL-6 levels were calculated using ELISA (Bioneovan Co., Ltd., Beijing, China) with an assay range between $3.4 \mathrm{U} / \mathrm{mL}-200 \mathrm{U} / \mathrm{mL}$.

\section{Statistical analysis}

The Kruskal-Wallis and Mann-Whitney U tests were used for the analysis of numerical independent data. Receiver operating characteristic (ROC) curves were set up to identify the cut off value for KL6 to differentiate patients with and without ILD, matching them with the Youden method $(13,26)$, which detects sensitivity and specificity. The following parameters were also calculated: estimation of the area under the ROC curve and its $95 \%$ confidence interval, sensitivity, specificity, positive and negative predictive values related to the cut off value. A multiple regression analysis was used to analyze dichotomous dependent variables. All statistical analyses were applied using SPSS for Windows 15 statistical software. A value of $p<0.05$ was statistically significant, a value of $\mathrm{p}<0.001$ was highly statistically significant, and a value of $\mathrm{p}>0.05$ was nonstatistically significant.

\section{RESULTS}

\section{Patient demographic and clinical characteristics}

The study population consisted of 40 patients with CTD, 20 of whom had ILD (12 mild, 8 
severe). Their data were compared to those of 20 healthy control subjects. In the control group the mean age was $12 \pm 2.4$ years, versus $13 \pm 2.2$ years in CTD only group and $11.9 \pm 3.5$ years in CTD+ILD group. CTD duration was $3.2 \pm 2.5$ in CTD only group and $2.6 \pm 1.2$ in CTD+ILD group. The ILD duration was 1 year (min 0 , max 3 years). A female predominance was reported in all groups (Table I). The common symptoms/ signs at presentation were dyspnea $(80 \%)$, cough (60\%, dry with no sleep interruption), exercise limitation $(60 \%)$, frequent respiratory infections $(50 \%)$, wheezing $(33.3 \%)$, tachypnea and chest wall retraction $(10 \%)$ and clubbing (10\%). Two of children with mild ILD were asymptomatic and diagnosed by HRCT (10\%). The symptoms/signs severity correlated positively with severity ILD, as assessed by clinical examination.

\section{Serum KL-6 estimation among different groups}

The median serum level of KL-6 was 52.3 $\mathrm{U} / \mathrm{mL}(\min 32.8$; $\max 62.4 \mathrm{U} / \mathrm{mL})$ in the control group, $56.7 \mathrm{U} / \mathrm{mL}$ (min 35.8; $\max$ $68.5 \mathrm{U} / \mathrm{mL}$ ) in the CTD only group, $72.2 \mathrm{U} /$ $\mathrm{mL}(\min 58.4 ; \max 100.5 \mathrm{U} / \mathrm{mL}$ ) in mild ILD group, and $102.7 \mathrm{U} / \mathrm{mL}$ ( $\min 76.1$; $\max$ $180.8 \mathrm{U} / \mathrm{mL}$ ) in the severe ILD group. The KL-6 levels were higher in the severe ILD, intermediate in mild ILD group and lower in the control groups (Table II).

\section{Comparison of PFT, 6MWT among the different groups}

The pulmonary function test reflected a restrictive lung problem with decreased lung compliance and lung volumes. CTD only group showed a forced expiratory volume in $1 \mathrm{~s}$ (FEV1) (\% predicted) of $101 \pm 7$, forced vital capacity (FVC) (\% predicted) of $95 \pm 7$, the mild ILD group showed a FEV1\% of $77 \pm 10, \mathrm{FVC} \%$ of $72 \pm 7$, and the severe ILD group showed a FEV $1 \%$ of $55 \pm 5$, FVC $\%$ of $49 \pm 8$, with significant differences between groups. In 6MWT, the mean end-test $\mathrm{SpO} 2$ was $96 \% \pm 3$ of CTD only group vs $92 \% \pm 2$, $67 \% \pm 5$ in mild ILD and severe ILD groups, respectively. The mean end-test dyspnea

Table I - Demographic and clinical characteristics of the study groups.

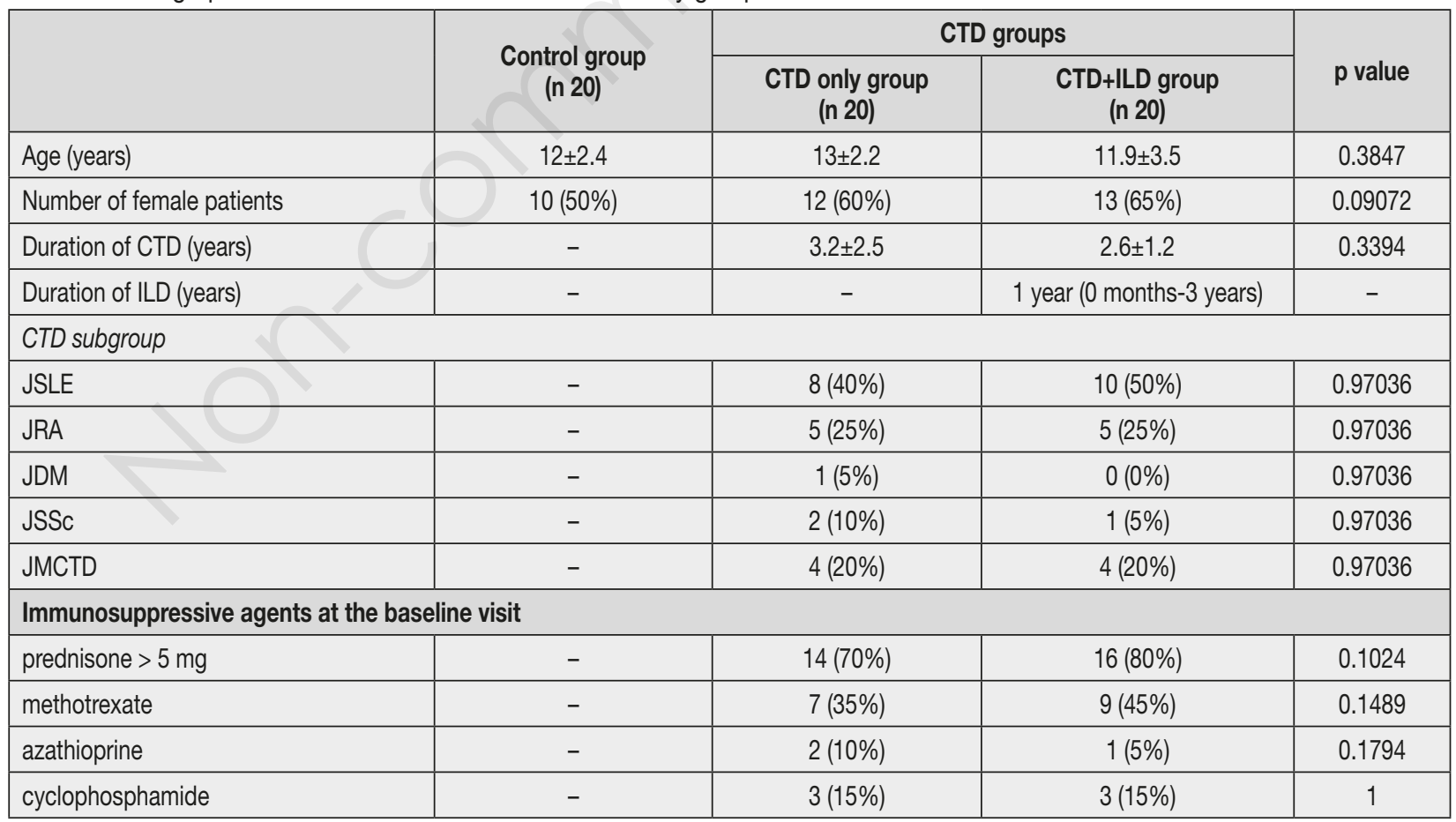

JRA, juvenile rheumatoid arthritis; JSLE, juvenile systemic lupus erythematosus; JSSc, juvenile systemic sclerosis; JDM, juvenile dermatomyositis; JMCTD, juvenile mixed connective tissue disease. One-way ANOVA, Chi-Square, and Paired sample T tests, $p>0.05$ : no significant differences, $p<0.05$ : significant differences, $p<0.001$ : highly significant differences. 
Table II - Comparison of KL-6 levels among groups.

\begin{tabular}{|l|c|c|c|}
\hline \multirow{2}{*}{} & \multicolumn{3}{|c|}{ KL-6 levels (U/mL) } \\
\cline { 2 - 4 } & Min-max & Median & p value \\
\hline Control group & $32.8-62.4$ & 52.3 & $<0.05^{\circledR, \#}$ \\
\hline CTD only group & $35.8-68.5$ & 56.7 & $<0.05^{\circledR, \#}$ \\
\hline Mild ILD subgroup & $58.4-100.5$ & 72.2 & $<0.05^{\star, \$, \#}$ \\
\hline Severe ILD subgroup & $76.1-180.8$ & 102.7 & $<0.05^{\star, \$, \Theta}$ \\
\hline
\end{tabular}

Kruskal-Wallis and Mann-Whitney U tests. *Significant differences vs control group, ${ }^{\circledR}$ significant differences vs CTD group, ${ }^{\circledR}$ significant differences vs mild ILD group), " significant differences vs severe ILD group).

level graded with the modified Borg scale was $2 \pm 1$ in the CTD only group vs $3 \pm 2,4 \pm 3$ in mild ILD and severe ILD groups, respectively (Table III).

\section{Associations between serum KL-6 level and spirometry results}

Figure 1 shows a significant inverse correlation between serum KL-6 levels and predicted FEV1 (\% Pred) ( $\mathrm{r}=-0.7056$, $\mathrm{p}=0.000$ ), and a significant inverse correlation between serum KL-6 levels and FVC (\%Pred) ( $\mathrm{r}=-0.7745, \mathrm{p}=0.000)$.

\section{Prediction of ILD by multiple regression analysis and ROC curve analysis}

Table IV showed that the most sensitive variable to detect ILD in CTD children was the serum KL-6 level and FVC\% of predicted value. The other variables were not significant in terms of ILD detection. According to the Receiver Operating Characteristic (ROC) curve analysis, the area under the curve was of 0.977 and the cut-off value of serum KL-6 to distinguish ILD was $63.4 \mathrm{U} /$ $\mathrm{mL}$, with sensitivity of $95.2 \%$, specificity of $89.7 \%$, positive predictive value of $83.26 \%$ and negative predictive value of $97.2 \%$ (Figure 2). Moreover, FVC\% Receiver Operating Characteristic (ROC) curve analysis showed that the area under the curve was of 0.979 and the cut-off value of FVC\% to distinguish ILD was 79 , with sensitivity of $95.2 \%$, specificity of $100 \%$, positive predictive value of $100 \%$ and negative predictive value of $95.4 \%$ (Figure 3). The comparison between KL6 and FVC\% ROC curves showed that there is no statistical difference ( $p=0.7385$ ) between KL6 and FVC\% ROC curves.

\section{DISCUSSION AND CONCLUSIONS}

Juvenile CTD is marked by high morbidity and mortality and can affect any organs. ILD is one of the most significant complication of CTD $(27,28)$. The serum KL-6 level is se-

Table III - Comparison of PFT, 6MWT among the different groups.

\begin{tabular}{|c|c|c|c|c|}
\hline \multirow{2}{*}{ Variable } & \multirow{2}{*}{$\begin{array}{l}\text { CTD only group } \\
(20 \mathrm{n} .)\end{array}$} & \multicolumn{2}{|c|}{ CTD+ILD group } & \multirow{2}{*}{$p$ value } \\
\hline & & Mild ILD (12 n.) & Severe ILD (8n.) & \\
\hline FVC, \% predicted & $95 \pm 7$ & $72 \pm 7^{*}$ & $49 \pm 8^{*, * \star}$ & 0.0000 \\
\hline FEV1, \% predicted & $101 \pm 7$ & $77 \pm 10^{*}$ & $55 \pm 5^{\star, * *}$ & 0.0000 \\
\hline${ }^{1} \mathrm{SpO} 2$ at start of the test, \% & $98 \pm 2$ & $95 \pm 3$ & $88 \pm 4^{*, \star *}$ & 0.0000 \\
\hline${ }^{2} \mathrm{SpO} 2$ at end of the test, $\%$ & $97 \pm 3$ & $92 \pm 2^{*}$ & $67 \pm 5^{\star, \star *}$ & 0.0000 \\
\hline$p$ value ${ }^{(1 \text { vs } 2)}$ & 0.2225 & 0.0086 & 0,0001 & \\
\hline${ }^{3}$ Dyspnea at start of the test & 0 & $1 \pm 0.5^{*}$ & $3 \pm 1^{\star, \star \star}$ & 0.0000 \\
\hline${ }^{4}$ Dyspnea at end of the test & $2 \pm 1$ & $3 \pm 2$ & $4 \pm 3^{*}$ & 0.0101 \\
\hline$p$ value ${ }^{(3 \text { vs } 4)}$ & 0.0001 & 0.0028 & 0.3862 & \\
\hline
\end{tabular}

One-way ANOVA, least significant difference (LSD), paired T test (before and after the test): $p>0.05$ : no significant differences, $p<0.05$ : significant differences, $p<0.001$ : highly significant differences, $\left({ }^{*} V_{s}\right.$ CTD only group, ${ }^{* \star} V s$ mild ILD group). 
creted normally by type II alveolar epithelium and abnormally increased in case of lung tissue regeneration (10-12). KL-6 can be considered as a sensitive and specific marker for the development of ILD (13-16). As a result of this review, our study aimed to evaluate the correlation between serum KL-6 lev- els with both the presence of ILD and its severity in children with connective tissue diseases to ensure early detection and better management of ILD in children with CTD. As to the associations between serum KL-6 levels and other spirometry pulmonary function parameters, this study determined

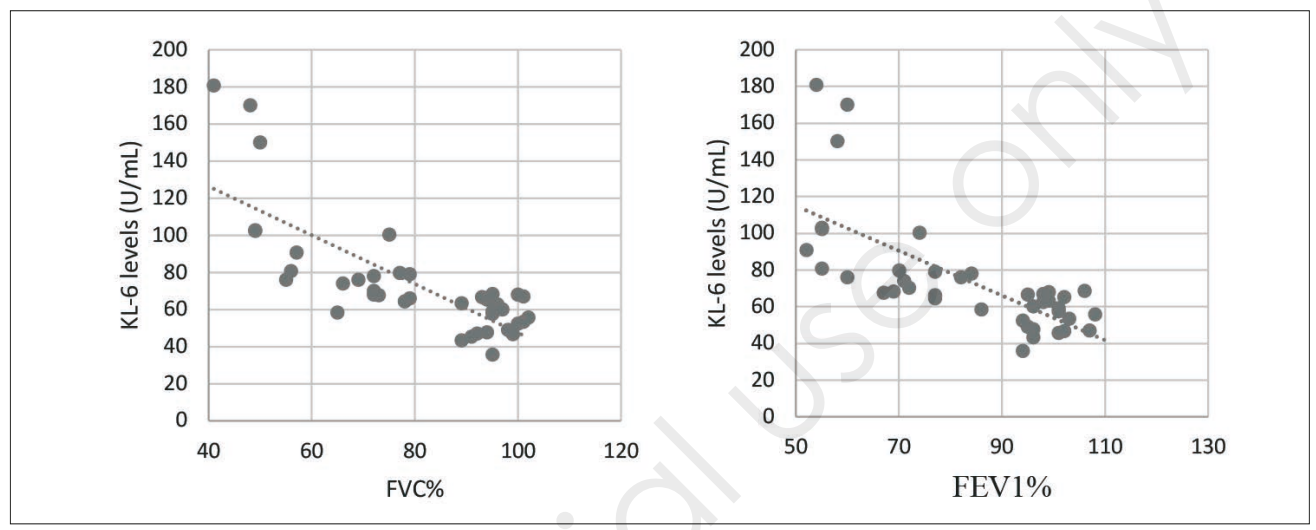

Figure 1 - Correlations between serum KL-6 levels and other PFT parameters.

Table IV - Prediction of ILD by multiple regression analysis.

\begin{tabular}{|l|c|c|c|c|}
\hline Variables & Coefficient & Std. Error & $\mathbf{t}$ & $\mathbf{p}$ \\
\hline Serum KL-6 & 0.1608 & 0.03694 & 4.354 & $0.0001^{*}$ \\
\hline FEV1\% of predicted & -0.02472 & 0.1107 & -0.223 & 0.8247 \\
\hline FVC\% of predicted & 0.3104 & 0.1313 & 2.363 & $0.0246^{*}$ \\
\hline Dyspnea at end of the test & 0.4410 & 0.4961 & 0.889 & 0.3808 \\
\hline SpO2 at end of the test & 0.1582 & 0.1271 & 1.244 & 0.2228 \\
\hline
\end{tabular}
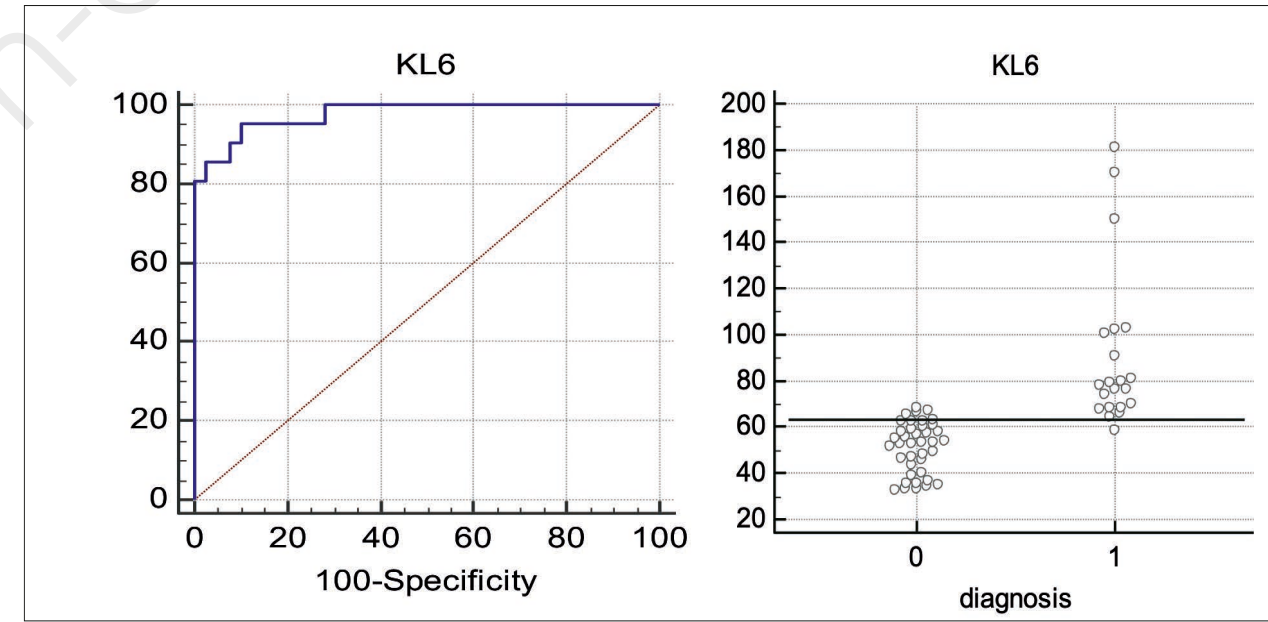

Figure 2 - KL-6 Receiver-Operating Characteristic (ROC) curve to diagnose CTD with interstitial lung disease, and the optimal cut off value (diagnosis: 0: controls and patients without ILD; 1 : patients with ILD). 

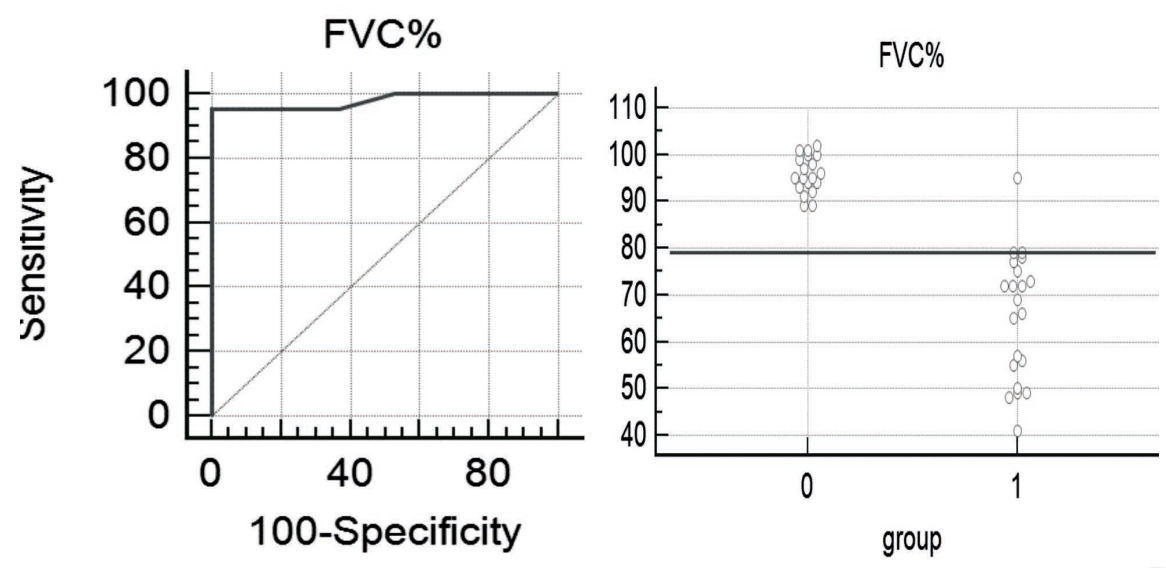

Figure 3 - FVC\% Receiver-Operating Characteristic (ROC) curve to diagnose CTD with interstitial lung disease, and the optimal cut off value (diagnosis: 0 : controls and patients without ILD; 1 : patients with ILD).

that there is a significant inverse correlation between serum KL-6 levels and all spirometry results: predicted FEV1 (\%Pred), and FVC (\%Pred). This was in agreement with Fathi et al., who reported a negative correlation between serum KL-6 level and spirometry results in patients with ILD (29). In another study, Hu et al. reported that the KL-6 levels were significantly inversely correlated with PFT (12). The same result was achieved by Xue et al. in a study on ILD associated with asbestosis and silicosis; these authors stated that serum KL-6 concentration was negatively correlated with predicted FVC\% (30).

Our multiple regression analysis showed that both KL-6 and FVC\% can be considered as indicators of ILD. According to the Receiver Operating Characteristic Curve (ROC) analysis for KL-6, the area under the curve was of 0.977 and the optimal cut-off value of serum KL-6 to discriminate the presence of ILD was $63.4 \mathrm{U} / \mathrm{mL}$, with sensitivity of $95.2 \%$, specificity of $89.7 \%$, positive predictive value of $83.26 \%$ and negative predictive value of $97.2 \%$, while the FVC ROC curve analysis showed that the area under the curve was of 0.979 and the optimal cut-off value of serum $\mathrm{FVC} \%$ to discriminate the presence of ILD was 79, with sensitivity of $95.2 \%$, specificity of $100 \%$, positive predictive value of $100 \%$ and negative predictive value of $95.4 \%$. This matched with results achieved by Kobayashi et al. who mentioned that KL-6 could discriminate between ILD and other common lung diseases. A cut-off of $500 \mathrm{U} / \mathrm{ml}$ was established to distinguish adult patients with ILD from healthy controls and those with other lung disorders (31). Also, Zheng et al. mentioned that the area under the curve for serum KL-6 was 0.911 , indicating a good diagnostic performance for idiopathic interstitial pneumonia, with sensitivity of $85.33 \%$, specificity of $90.00 \%$, positive predictive value of $95.52 \%$, negative predictive value of $71.05 \%$, and Kappa value of 0.70 (32). Furthermore, KL-6-ROC curve analysis by Lee et al. showed that $275.1 \mathrm{U} / \mathrm{mL}$ was the serum KL-6 concentration for detecting the presence of ILD in CTD patients (sensitivity 79.4\%, specificity 79.9\%) (33). Pairwise comparison of ROC curves showed that there is no statistical difference between KL6 and FVC\% ROC curves, but KL-6 is more valuable in early detection of mild patients, easy to measure with routine blood investigations, and does not require any cooperation from the patient, particularly in pediatric cases.

Furthermore, Ishikawa et al. mentioned that patients with ILD had abnormal KL-6 levels, as opposed to only $10 \%$ of patients with pneumonia, asthma, or chronic obstructive pulmonary disorder and $28 \%$ of patients with active pulmonary tuberculosis (34). 
This means that KL-6 is more specific for ILD than FVC. Our conclusion agrees with the results reported by Salazar et al., who stated that KL-6 was predictive of early SSc-ILD progression and higher KL-6 levels were predictive of faster FVC\% decline at the 1-year follow up (11). This is also in agreement with Fukaya et al. who reported that the KL-6 level should be sequentially measured in CTD patients, when interstitial lung diseases develop (35). Moreover, Hu et al. (12) showed that post-treatment serum KL-6 levels were reduced in patients with improved ILDs and were increased in patients with advanced ILDs, thus showing the importance of KL-6 level as a prognostic tool and for treatment monitoring purposes. As to the comparison of KL- 6 and HRCT for the early detection of ILD, we agree with Lee at al., who reported that regular chest HRCT in patients with these CTDs is not currently recommended. Considering cost-effectiveness and radiation hazard, KL-6 measurement using a simple blood test would be a good alternative to chest HRCT for evaluating the current status of ILD, regardless of the CTD type. Furthermore, chest HRCT can provide objective evidence of exacerbation with overt clinical symptoms, but the optimal time interval between CT scans in asymptomatic ILD patients is difficult to determine. Regular chest HRCT, for instance yearly, combined with more frequent KL-6 measurements could be an ideal protocol for detecting worsening CTD-ILD (33).

\section{Acknowledgements}

We wish to express our deepest gratefulness to all nurse staff in the Pulmonology, Immunology and Allergy Units, at the children hospital, Zagazig University and to all patients and their family for patience and moral support for the completion of this work.

\section{Conflict of interest}

The authors declare no potential conflicts of interest.

\section{REFERENCES}

1. Cojocaru M, Cojocaru IM, Silosi I, Vrabie CD. Pulmonary manifestations of systemic autoimmune diseases. Maedica. 2011; 6: 224-229.
2. Quezada A, Ramos S, Garcia M, et al. Lung involvement in rheumatologic diseases in children. ALLER Allergol Immunopathol. 2012; 40: 88-91.

3. Shahane A. Pulmonary hypertension in rheumatic diseases: epidemiology and pathogenesis. Rheum Int (Berlin). 2013; 33: 1655-1667.

4. Ahuja J, Arora D, Kanne JP, et al. Imaging of Pulmonary Manifestations of Connective Tissue Diseases. RCL Radiol Clin N Am. 2016; 54: 1015-1031.

5. Yun D, Stein SL. Review of the cutaneous manifestations of autoimmune connective tissue diseases in pediatric patients. WJD World $\mathbf{J}$ Dermatol. 2015; 4: 80.

6. Cidon M, Bansal M, Hartl D. Pulmonary manifestations of rheumatologic diseases. Curr Opin Pediatr. 2017; 29: 311-319.

7. Solomon JJ, Fischer A. Connective tissue diseaseassociated interstitial lung disease: a focused review. J Intens Care Med. 2015; 30: 392-400.

8. Saper VE, Chen G, Deutsch GH, et al. Emergent high fatality lung disease in systemic juvenile arthritis. Ann Rheum Dis. 2019; 78: 1722-1731.

9. Schulert GS, Yasin S, Carey B, et al. Systemic juvenile idiopathic arthritis-associated lung disease: characterization and risk factors. Arthritis Rheumatol. 2019; 71: 1943-1954.

10. Kumanovics G, Gorbe E, Minier T, et al. Follow-up of serum KL-6 lung fibrosis biomarker levels in 173 patients with systemic sclerosis. Clin Exp Rheumatol. 2014; 32: S138-S144.

11. Salazar GA, Kuwana M, Wu M, et al. KL-6 But Not CCL-18 is a predictor of early progression in systemic sclerosis-related interstitial lung disease. J Rheumatol. 2018; 45: 1153-1158.

12. Hu C, Wu C, Yang E, et al. Serum KL-6 is associated with the severity of interstitial lung disease in Chinese patients with polymyositis and dermatomyositis. Clin Rheumatol. 2019; 38: 2181-2187.

13. Benyamine A, Heim X, Resseguier N, et al. Elevated serum Krebs von den Lungen-6 in systemic sclerosis: a marker of lung fibrosis and severity of the disease. Rheumatol Int. 2018; 38: 813-819.

14. Hanaoka M, Katsumata Y, Kawasumi H, et al. KL-6 is a long-term disease-activity biomarker for interstitial lung disease associated with polymyositis/dermatomyositis, but is not a shortterm disease-activity biomarker. Modern Rheumatol. 2019; 29: 625-632.

15. Hu Y, Wang LS, Jin YP, et al. Serum Krebs von den Lungen-6 level as a diagnostic biomarker for interstitial lung disease in Chinese patients. Clin Respir J. 2017; 11: 337-345.

16. Kilinc AA, Arslan A, Yildiz M, et al. Serum KL-6 level as a biomarker of interstitial lung disease in childhood connective tissue diseases: a pilot study. Rheumatol International. 2019 [Epub ahead of print). 
17. Aletaha D, Neogi T, Silman AJ, et al. 2010 Rheumatoid Arthritis Classification Criteria An American College of Rheumatology/European League Against Rheumatism Collaborative Initiative. Arthrit Rheum. 2010; 62: 2569-2581.

18. Aringer M, Costenbader K, Daikh D, et al. 2019 European League Against Rheumatism/ American College of Rheumatology classification criteria for systemic lupus erythematosus. Ann Rheum Dis. 2019; 78: 1151-1159.

19. Lundberg IE, Tjärnlund A, Bottai M, et al. 2017 European League Against Rheumatism/American College of Rheumatology classification criteria for adult and juvenile idiopathic inflammatory myopathies and their major subgroups. Ann Rheum Dis. 2017; 76: 1955-1964.

20. Zulian F, Woo P, Athreya BH, et al. The Pediatric Rheumatology European Society/American College of Rheumatology/European League against Rheumatism provisional classification criteria for juvenile systemic sclerosis. ART Arthrit Care \& Res. 2007; 57: 203-212.

21. Tanaka Y, Kuwana M, Fujii T, et al. 2019 Diagnostic criteria for mixed connective tissue disease (MCTD): From the Japan research committee of the ministry of health, labor, and welfare for systemic autoimmune diseases. Modern Rheumatol. 2020; 1-5.

22. Raghu G, Remy-Jardin M, Myers JL, et al. Diagnosis of idiopathic pulmonary fibrosis. An Official ATS/ERS/JRS/ALAT Clinical Practice Guideline. Am J Respir Crit Care Med. 2018; 198: e44-e68.

23. Kuo CS, Young LR. Interstitial lung disease in children. Curr Opin Pediatr. 2014; 26: 320-327.

24. Jiang Z, Tao JH, Zuo T, et al. The correlation between miR-200c and the severity of interstitial lung disease associated with different connective tissue diseases. Scand J Rheumatol. 2017; 46: 122-129.

25. Lancaster LH. Utility of the six-minute walk test in patients with idiopathic pulmonary fibrosis. Multidiscip Respir Med. 2018; 13: 45.
26. Shapiro DE. The interpretation of diagnostic tests. Stat Methods Med Res. 1999; 8: 113-134.

27. Satoh H, Kurishima K, Ishikawa H, Ohtsuka M. Increased levels of KL-6 and subsequent mortality in patients with interstitial lung diseases. J Intern Med. 2006; 260: 429-434.

28. Oguz EO, Kucuksahin O, Turgay M, et al. Association of serum KL-6 levels with interstitial lung disease in patients with connective tissue disease: a cross-sectional study. Clin Rheumatol. 2016; 35: 663-666.

29. Fathi M, Barbasso Helmers S, Lundberg IE. KL-6: a serological biomarker for interstitial lung disease in patients with polymyositis and dermatomyositis KL-6: a serological biomarker for interstitial lung disease. J Intern Med. 2012; 271: 589-597.

30. Xue C, Wu N, Li X, et al. Serum concentrations of Krebs von den Lungen-6, surfactant protein $\mathrm{D}$, and matrix metalloproteinase- 2 as diagnostic biomarkers in patients with asbestosis and silicosis: a case-control study. BMC Pulm Med. 2017; 17: 144.

31. Kobayashi J, Itoh Y, Kitamura S, Kawai T. [Establishment of reference intervals and cut-off value by an enzyme immunoassay for KL-6 antigen, a new marker for interstitial pneumonia]. Rinsho Byori. 1996; 44: 653-658.

32. Zheng P, Liu X, Huang H, et al. Diagnostic value of KL-6 in idiopathic interstitial pneumonia. J Thorac Dis. 2018; 10: 4724-4732.

33. Lee J, Lee Y, Ha Y, et al. Serum KL-6 levels reflect the severity of interstitial lung disease associated with connective tissue disease. Arthrit Res Ther. 2019; 21: 1-8.

34. Ishikawa N, Hattori N, Yokoyama A, Kohno N. Utility of KL-6/MUC1 in the clinical management of interstitial lung diseases. Respir Investig. 2012; 50: 3-13.

35. Fukaya $S$, Oshima $H$, Kato $K$, et al. KL-6 as a novel marker for activities of interstitial pneumonia in connective tissue diseases. Rheumatol Int. 2000; 19: 223. 